\title{
Polymorphism rs7521584 in miR-429 is associated with the severity of atrophic gastritis in patients with Helicobacter pylori infection
}

\author{
TOSHIMI OTSUKA ${ }^{1}$, TOMOMITSU TAHARA ${ }^{2}$, MASAKATSU NAKAMURA ${ }^{1}$, WU JING ${ }^{1}$, MASAFUMI OTA ${ }^{1}$, \\ TOMOE NOMURA ${ }^{1}$, RANJI HAYASHI ${ }^{1}$, TAKEO SHIMASAKI ${ }^{1}$, TOMOYUKI SHIBATA ${ }^{2}$ and TOMIYASU ARISAWA ${ }^{1}$ \\ ${ }^{1}$ Department of Gastroenterology, Kanazawa Medical University, Uchinada-machi, Ishikawa 920-0293; \\ ${ }^{2}$ Department of Gastroenterology, Fujita Health University, Toyoake, Aichi 470-1192, Japan
}

Received August 29, 2016; Accepted March 1, 2017

DOI: $10.3892 / \mathrm{mmr} .2018 .9200$

\begin{abstract}
The aim of the present study was to investigate an association of genetic polymorphism (rs7521584) located in miR-200a-200b-429 cluster, which has tumor suppressor and pro-inflammatory function, with the development of gastric mucosal atrophy and metaplasia as a pre-malignant condition. Gastric mucosa samples were obtained from the antrum of 393 patients with no malignancies. The rs7521584 genotype was determined using the polymerase chain reaction-single-strand conformation polymorphism analysis method. The degree of gastritis was assessed histologically in all subjects and serum levels of pepsinogen (PG) I/II were quantified in 123 out of 393 patients. Patients with an atrophy score $\geq 1$ and metaplasia score $\geq 1$ were classified into the atrophic gastritis group (AG group). The rs7521584 TT genotype was significantly associated with the development of atrophic gastritis [odds ratio (OR), 2.41; $95 \%$ confidence interval $(\mathrm{CI}), 1.10-5.25 ; \mathrm{P}=0.027)$, particularly in patients with $H$. pylori infection (OR, 3.31; 95\% CI, 1.35-8.12; $\mathrm{P}=0.0089)$. In addition, in patients younger than 60 years of age, this genotype was associated with atrophic gastritis (OR, 3.15; 95\% CI 1.03-9.61; $\mathrm{P}=0.044)$ ]. In patients with $H$. pylori infection, the metaplasia score was significantly higher in the TT homozygote compared with the GG+GT genotype. In the rs7521584 TT homozygote, serum PG I/II ratio was significantly reduced with increasing age $(\mathrm{P}=0.0084)$. No significant trend was identified between the GG+GT genotype and age. The results of the current study indicated that the rs7521584 minor allele homozygote was associated with the development of chronic gastritis under the influence of $H$.pylori-induced inflammation, particularly with the severity of metaplastic alterations.
\end{abstract}

Correspondence to: Dr Tomiyasu Arisawa, Department of Gastroenterology, Kanazawa Medical University, 1-1 Daigaku, Uchinada-machi, Ishikawa 920-0293, Japan

E-mail: tarisawa@kanazawa-med.ac.jp

Key words: miR-200 family, miR-429, rs7521584, atrophic gastritis, Helicobacter pylori infection

\section{Introduction}

Gastric cancer remains to be the third leading cause of cancer mortality, worldwide; however, the incidence rates have declined in Western Europe (1). Helicobacter pylori (H.pylori) infection is regarded as a primary factor in the development of atrophic gastritis, and gastric cancer may subsequently occur $(2,3)$. Gastric cancer occurs via numerous stages, beginning with chronic inflammation, atrophic alterations, intestinal metaplasia and dysplasia (4). From the majority of patients with an $H$. pylori infection, only a small percentage of patients have the poorest clinical outcome, such as diagnosis with gastric cancer. However, a specific factor which may aid in determining the variation of the clinical outcome remains to be identified, the specific outcome may be the result of an interaction of bacterial virulence factors, host immune system and the environment. Additionally, a previous study determined that genetic variation affected the clinical course of $H$. pylori infection (5).

A microRNA (miRNA) is an endogenous small non-protein-coding RNA cleaved from a precursor of the 70-100 bp hairpin form $(6,7)$. miRNAs, which bind to the 3'-untranslated region (UTR) of target genes, regulate the mRNA expression post-transcriptionally (6). Previous studies which examined expression of miRNAs using clinical samples, determined that the expression and function of miRNAs may be associated with cancer pathogenesis (8-10) including gastric cancer (11). Mishra et al (12) determined that polymorphism of pri-, pre- and mature miRNA may affect miRNA function and potentially influence various gene expression levels and signaling pathways. It has also been reported that the processing and target selection of miRNAs may be influenced by variations in the coding region of miRNA genes, including pri-, pre- and mature miRNAs (13). The authors of the present study previously reported that miR-27a genetic variation may be associated with gastric mucosal atrophy (14).

The present study, investigated the region of $\sim 2 \mathrm{kbp}$ at chromosome 1, containing miRNA (miR)-200b, miR-200a and miR-429 (Fig. 1). From this region, 5 miRNAs are yielded and may regulate various gene expression levels. It is possible that genetic variations in this region may influence the clinical outcome of some diseases. The rs7521584 polymorphism 
reported in National Center for Biotechnology Information single nucleotide polymorphism (SNP) database was selected, and the present study investigated the association of this polymorphism with the development of gastric mucosal atrophy and metaplastic change as a pre-malignant condition.

\section{Materials and methods}

Clinical samples and DNA extraction. A total of 393 patients with no neoplastic lesions, who had a medical examination in Fujita Health University Hospital (Toyoake, Japan) or Kanazawa Medical University Hospital (Uchinada-machi, Japan) from April 2005 to March 2014 were recruited for the present study. All patients underwent upper gastrointestinal endoscopy due to reported abdominal discomfort and were diagnosed with gastritis or no abnormal appearance. Patients with severe systemic diseases, malignancies in other organs and those that had received nonsteroidal anti-inflammatory drugs, antibiotics and $H$.pylori eradication treatment were excluded.

All histological diagnoses were preformed using biopsy specimens obtained from the antrum by the Division of Pathology of each hospital. The severity of chronic gastritis was classified according to the updated Sydney system (15) by a pathologist who had no access to any clinical information. According to the severity of atrophic gastritis, the subjects were divided into the following 2 groups: The atrophic gastritis (AG) group (atrophy score $\geq 1$ and metaplasia score $\geq 1$ ) and the no atrophy (NA) group.

Genomic DNA was isolated from the blood samples using QIAamp DNA blood kit (Qiagen, Inc., Valencia, CA, USA) according to the manufacturer's protocol. H. pylori infection status was determined by serology, histological examination and the urea breath test. A total of 251 patients were diagnosed as $\mathrm{H}$. pylori positive when at least one of the diagnostic tests was positive. In addition, serum pepsinogen (PG) I/II levels were quantified in 123/393 patients.

The Ethical Committee of Fujita Health University and Kanazawa Medical University approved the protocol and written informed consent was obtained from all patients.

SNP detection. In order to determine the genotype of patients, the present study used polymerase chain reaction-single strand conformation polymorphism (PCR-SSCP) as previously described (14). Primer pairs were synthesized and the sequences were as follows: rs7521584 forward, 5'-AACAGTGGCCTC TCTCACGTGGT-3' and reverse, 5'-TTGCAGATGGAAAAG ATGAAACAAT-3'. PCR was performed in a volume of $20 \mu 1$ containing $0.1 \mu \mathrm{g}$ DNA using Ex Taq DNA polymerase (Takara Bio Inc., Otsu, Japan). The DNA was denatured at $95^{\circ} \mathrm{C}$ for $3 \mathrm{~min}$, followed by 35 cycles at $95^{\circ} \mathrm{C}$ for $30 \mathrm{sec}, 54^{\circ} \mathrm{C}$ for $40 \mathrm{sec}$ and $72^{\circ} \mathrm{C}$ for $45 \mathrm{sec}$, with a final extension step at $72^{\circ} \mathrm{C}$ for $5 \mathrm{~min}$. Subsequently, $2 \mu 1$ of the PCR products were denatured using $10 \mu \mathrm{l}$ formamide (Sigma-Aldrich; Merck Millipore, Darmstadt, Germany) at $90^{\circ} \mathrm{C}$ for $5 \mathrm{~min}$. SSCP was performed at $18^{\circ} \mathrm{C}$ using a GenePhor DNA separation system with GeneGel Excel 12.5/24 (GE Healthcare Life Sciences, Chalfont, UK), subsequently the denatured single strand DNA bands were detected using a DNA Silver Staining kit (GE Healthcare Life Sciences).

Reverse transcription-quantitative PCR (RT-qPCR). Total RNA from whole blood samples of healthy patients with $5 \mathrm{GG}$,

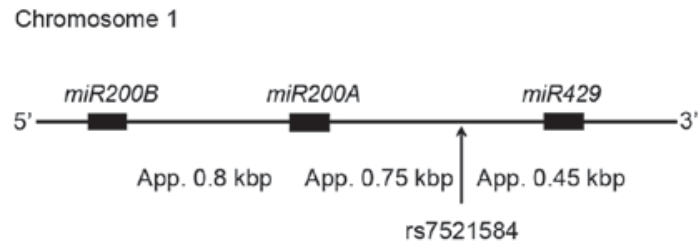

Figure 1. rs7521584 is located between MIR429 and MIR200s on chromosome 1 . A total of 5 miRNAs were identified, miR200B-5p, miR200B-3p, miR200A-5p, miR200A-3p and miR429.

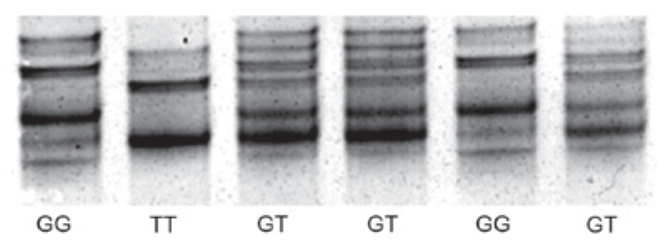

Figure 2. Images of polymerase chain reaction-SSCP. Single strand DNAs of rs7521584 genotype were clearly separated by SSCP. SSCP, single strand conformation polymorphism.

5GT and 5TT genotypes was extracted using TRIzol reagent (Thermo Fisher Scientific, Inc., Waltham, MA, USA) according to the manufacturer's instructions. The Mir-X miRNA qRT-PCR SYBR kit (Takara Bio, Inc.) was used to quantify miRNA expression, according to the manufacturer's instructions. Total RNA $(0.8 \mu \mathrm{g})$ was reverse transcribed using the Mir- $\mathrm{X}^{\mathrm{TM}}$ miRNA First-Strand Synthesis kit according the manufacturer's protocol. The PCR reactions were performed using miRNA-specific primers (miR-429, 5'-TAATACTGTCTGGTA AAACCGT-3'; miR-200a-3p, 5'-TAACACTGTCTGGTAACG ATGT-3' for miR-200a-3p). The thermocycling conditions were as follows: initial denaturation at $95^{\circ} \mathrm{C}$ for $10 \mathrm{sec}$, followed by 40 cycles of $95^{\circ} \mathrm{C}$ for $5 \mathrm{sec}$ and $60^{\circ} \mathrm{C}$ for $20 \mathrm{sec}$. U6 was used as the reference gene (5'-GGGCAGGAAGAGGGCCTAT-3'). All data were analyzed using the $2^{-\Delta \Delta \mathrm{Cq}}$ method (16).

Statistical analysis. The Hardy-Weinberg equilibrium of the allele was assessed using a $\chi^{2}$ test. The age data were expressed as the mean \pm standard deviation. Analyses were performed using Stata (version 13; StataCorp LP, College Station, TX, USA). The mean ages between the 2 groups were compared using Student's t-test. The ratios of $H$.pylori infection status and gender were compared using Fisher's exact test. Differences of genotype frequencies were determined with a two-sided Fisher's exact test. The odds ratio (OR) and 95\% confidence intervals (CI) were estimated by logistic regression following an adjustment for age, gender and $H$. pylori infection status. Each of the Sydney system scores between the 2 groups was compared using a Mann-Whitney U test. The association between age and PG I/II ratio was determined using one-way analysis of variance. $\mathrm{P}<0.05$ was considered to indicate a statistically significant difference.

\section{Results}

Characteristics of subjects and genotype frequencies. Single strand DNA of rs7521584 was clearly separated by SSCP (Fig. 2). A total of 393 patients participated in the present 
Table I. Characteristics of the subjects and frequency of genotypes.

\begin{tabular}{|c|c|c|c|c|}
\hline Characteristic & Total & NA group & AG group & P-value \\
\hline Number of patients & 393 & 254 & 139 & \\
\hline Mean age \pm standard deviation & $60.0 \pm 13.3$ & $58.0 \pm 13.7$ & $63.6 \pm 11.8$ & $<0.0001^{\mathrm{a}}$ \\
\hline Male:female & $233: 160$ & $132: 122$ & 101:38 & $<0.0001^{\mathrm{a}}$ \\
\hline H. pylori positive rate & $251 / 393$ & $118 / 254$ & $133 / 139$ & $<0.0001^{\mathrm{a}}$ \\
\hline \multicolumn{5}{|l|}{ rs7521584 G>T } \\
\hline GG & 139 & 88 & 51 & \\
\hline GT & 203 & 137 & 66 & \\
\hline $\mathrm{TT}$ & 51 & 29 & 22 & \\
\hline $\mathrm{T}$ allele frequency & $38.8 \%$ & $38.4 \%$ & $39.6 \%$ & NS \\
\hline
\end{tabular}

${ }^{a} \mathrm{NA}$ group vs. AG group. NA, no atrophy; AG, atrophic gastritis.
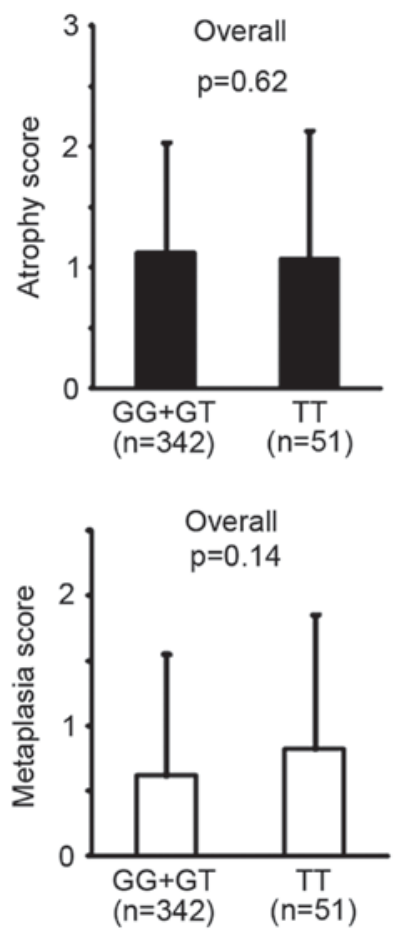
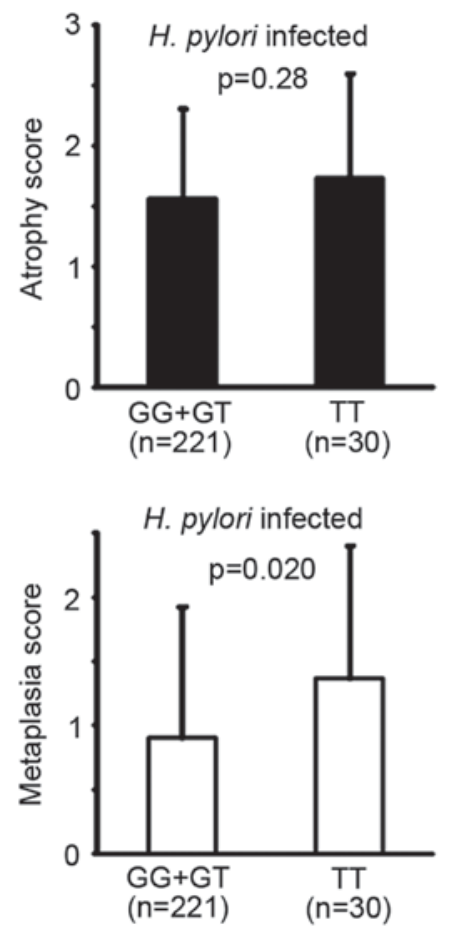

Figure 3. Comparison of atrophy and metaplasia scores between the GG+GT genotype and TT homozygote. In the $H$. pylori-infected subject, the metaplasia score was significantly higher in the TT homozygote compared with the GG+GT genotype.

study. The patients were classified into the AG $(n=139)$ and NA groups $(n=254)$, their characteristics are summarized in Table I. The mean age of the AG group was significantly greater compared with the NA group. The male/female ratio and $H$. pylori positivity were also significantly greater in the AG group compared with the NA group.

The distribution of genotypes in the patients recruited in the present study were in Hardy-Weinberg equilibrium $(\mathrm{P}=0.09)$. No significant difference between the genotype distribution of AG and NA groups was identified (Table I).

Association between the rs 7521584 polymorphism and atrophic gastritis. The present study used logistic regression analysis following an adjustment for age, gender and $H$. pylori infection status, to determine that the rs7521584 TT genotype was significantly associated with the severity of atrophic gastritis (OR, 2.41; 95\% CI, 1.10-5.25; P=0.027; Table II). In the H. pylori infected subjects, TT genotype was also significantly associated with the atrophic gastritis (OR, 3.31; 95\% CI, 1.35-8.12; P=0.0089; Table II). In addition, in the subjects younger than 60 years of age, this genotype was positively associated with atrophic gastritis (OR, 3.15; 95\% CI, 1.03-9.61; $\mathrm{P}=0.044$; Table II).

No significant difference was identified between the GG+GT genotype and TT homozygote for the atrophy and metaplasia scores in the updated Sydney system (Fig. 3). However, in patients with an $H$. pylori infection, the metaplasia score was significantly higher in the TT homozygote compared with the GG+GT genotype. 
Table II. Association between rs7521584 and gastric mucosal atrophy.

\begin{tabular}{|c|c|c|c|c|c|c|}
\hline Characteristic & No. of subjects & GG & GT & $\mathrm{TT}$ & OR $(95 \% \mathrm{CI})^{\mathrm{a}}$ & P-value \\
\hline \multicolumn{7}{|l|}{ rs7521584G>T } \\
\hline NA & 254 & 88 & 137 & 29 & Reference & - \\
\hline AG & 139 & 51 & 66 & 22 & $2.41(1.10-5.25)$ & $0.027^{\mathrm{a}}$ \\
\hline \multicolumn{7}{|c|}{ H. pylori positive } \\
\hline NA & 118 & 40 & 70 & 8 & Reference & - \\
\hline AG & 133 & 49 & 62 & 22 & $3.31(1.35-8.12)$ & $0.0089^{\circ}$ \\
\hline \multicolumn{7}{|l|}{$\leq 60$} \\
\hline NA & 129 & 48 & 64 & 17 & Reference & - \\
\hline AG & 46 & 13 & 23 & 10 & $3.15(1.03-9.61)$ & $0.044^{\mathrm{a}}$ \\
\hline
\end{tabular}

${ }^{a}$ TT vs. GG+GT. NA, no atrophy; AG, atrophic gastritis; CI, confidence interval.
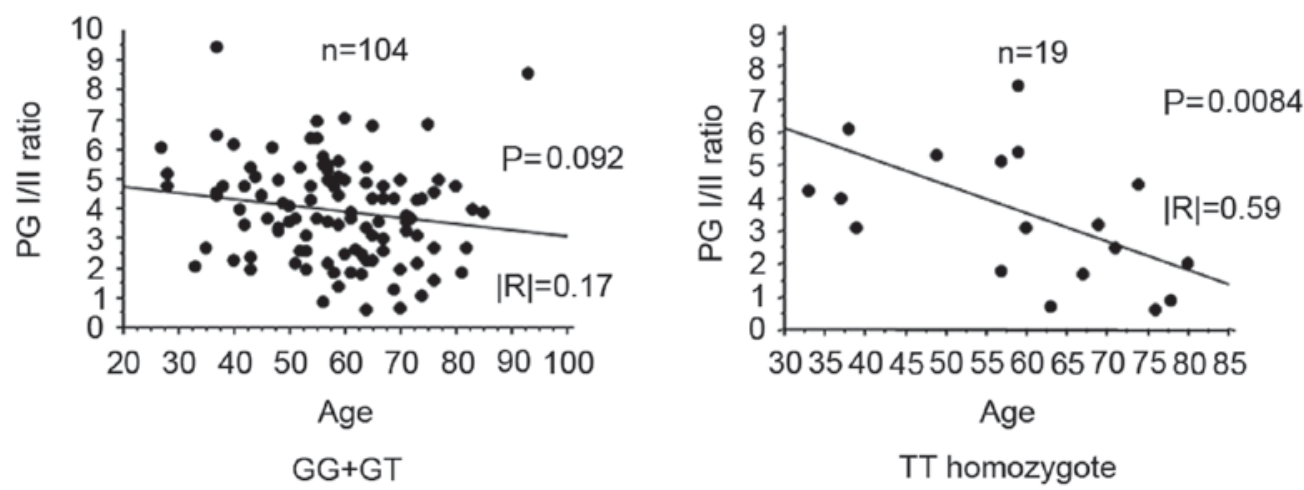

Figure 4. The serum PG I/II ratio and rs7521584 genotype. In the TT homozygote, serum PG I/II ratio was negatively correlated with age in the TT homozygote. $\mathrm{PG}$, pepsinogen.

Serum PG I/II ratio and rs7521584 genotype. The rs7521584 genotype distribution in 123 patients whose serum PG were quantified was as follows: GG, 47; GT, 57; and TT, 19, which did not differ from the distribution observed in all patients ( $\mathrm{P}=0.63)$. The H. pylori positivity did not differ significantly between the GG+GT genotype (61.5\%) and TT homozygote $(52.6 \%)$.

In the rs7521584 TT homozygote, PG I/II ratio significantly decreased with increasing age $(\mathrm{P}=0.0084$; Fig. 4). However, there was no significant association between the PG I/II ratio and age in the GG+GT genotype (Fig. 4).

\section{Discussion}

A total of five miRNAs with similar seed sequence constitute the miR-200 family, located in two distinct genomic clusters (miR-200a-200b-429 and miR-200c-141 clusters). The miR-200a-200b-429 cluster is located on chromosome 1 . In general, one mRNA is regulated by numerous miRNAs, and one miRNA may target numerous mRNAs (17). Therefore, it is difficult to determine the mechanisms of miRNA in the pathogenesis of human disorders. However, several previous studies have indicated that the reduced expression of miR-200 family may contribute to the development and progression of gastric cancer (18-20). Chang et al (18) determined that the expression of the miR-200 family was downregulated in gastric cancer tissues compared with matched non-cancerous tissues and lower levels of the miR-200 family were associated with the prognosis of the patients with gastric cancer (18). Zhu et al (19) has reported that suppressed expression of miR-429 in the gastric cancer cell line AGS promoted cancer cell survival mediated by B cell leukemia/lymphoma 2 against chemotherapy-induced cell death and that the rescued expression of miR-429 induced the apoptosis of cancer cells (19). Zhang et al (20) have indicated that expression of miR-429 in gastric cancer tissues was downregulated compared with adjacent normal tissue and have demonstrated that the expression of fascin-1 (a direct target of miR-429) and miR-429 expression were inversely correlated in vivo (20). These previous findings suggest that the miR-200 family may act as a tumor suppressor on the development and progression of gastric cancer. Additionally, the miR-200 family may also be capable of inhibiting the invasion of carcinoma to the other organs, including colorectal carcinoma (21-23). Conversely, Chen et al (24) reported that the overexpression of miR-200a-3p may be associated with gender and miR-429 with age in patients with gastric cancer. In addition, it has been previously reported that upregulated expression of miR-429 in patients with serous ovarian carcinoma was negatively correlated with survival (25). Therefore, although the miR-200 family may have important roles in tumor progression, their 
specific functions and the mechanisms involved remain to be fully elucidated.

The miR-200 family also has an important role in the inflammation process. Reddy et al (26) identified a proinflammatory function for the negative feedback loop between miR-200 and zinc finger e-box-binding homeobox 1 in vascular smooth muscle cells (VSMCs) under diabetic conditions (26). They concluded that disruption of this negative feedback loop enhances the proinflammatory responses of VSMCs, which are implicated in vascular complications (26). Xiao et al (27) have determined that miR-429 targets dual specificity protein phosphatase 1 directly, in order to regulate the activation of p38 mitogen-activated protein kinase and subsequent production of cytokines in response to lipopolysaccharide stimulation in alveolar macrophages (27). The miR-200 family may have a tumor suppressor role by inhibition of the epithelial mesenchymal transition (28) and a proinflammatory role in the inflammation process.

In the present study, rs7521584 was significantly associated with the development of atrophic gastritis, which is considered as a pre-malignant condition, particularly in patients with H. pylori infection. Additionally, the significant progression of atrophic gastritis was observed in patients younger than 60 years of age. Although no difference was identified in terms of $H$. pylori positivity between the two genotypes, serum PG I/II ratio was reduced with age in the TT homozygote compared with the GG+GT genotype. The present results suggest that $H$.pylori-associated atrophic gastritis may progress more rapidly in the TT homozygote compared with the other genotypes. Additionally, the present study indicated that re7521584 is more closely associated with metaplastic alterations compared with atrophic alterations. A previous study indicated that the development of gastric metaplasia may not always be associated with the progression of gastric mucosal atrophy and $H$. pylori infection status (29). Gastric glandular atrophy, resulting from parietal cell loss due to $H$. pylori induced-inflammation, may lead to gastric chief cell transdifferentiation into spasmolytic polypeptide expressing metaplasia (SPEM) (30). In the presence of chronic inflammation, SPEM may progress to more advanced metaplasia but not to intestinal metaplasia in mice $(31,32)$. However, in humans, SPEM leads to intestinal metaplasia, which may progress to cancer (33). Therefore, it is likely that more advanced factors are required to develop intestinal metaplasia from atrophic gastric mucosa in humans that have yet to be identified (30). The rs7521584 polymorphism may be more closely associated with metaplastic transformation than atrophic change by $H$. pylori-induced chronic inflammation.

The contents of miR-200 families in blood samples were quantified and no significant differences were identified among rs7521584 genotypes (data not shown). Therefore, the influence of rs7521584 on the expression or function of miR-200 families cannot be evaluated. miRNAs may be derived by various stimulations and the content of miRNA in blood serum may differ from that in organ tissues, its influence may be difficult to determine solely from the quantification of the serum sample. Further investigation using cell assays would be required at this point. If variation of rs7521584 may influence the expression or function of one or all miR-200 families; therefore, rs7521584 may influence the metaplastic transformation in the gastric mucosa, leading to the later intestinal type of gastric cancer, by affecting mRNA expression via the alteration of miR-200 family expression or function.

In conclusion, the present study demonstrated that the rs7521584 minor allele homozygote was associated with the development of atrophic gastritis following $H$. pylori-induced inflammation, particularly affecting the severity of metaplastic alterations. The accumulation of similar findings may lead to an improved understanding of the diverse clinical course of H. pylori infection.

\section{Acknowledgements}

Not applicable.

\section{Funding}

Not applicable.

\section{Availability of data and materials}

The analyzed datasets generated during the study are available from the corresponding author on reasonable request.

\section{Author's contributions}

TA analyzed the data and was responsible for the conception of the study and designed the study. TO analyzed the data and wrote the paper. MN, MO, TN and RH obtained the samples and the data. WJ and TaS determined the genotypes. TT and ToS participated in the design of the study.

\section{Ethics approval and consent to participate}

TheEthicalCommittee ofFujitaHealthUniversity and Kanazawa Medical University approved the protocol and written informed consent was obtained from all patients.

\section{Consent for publication}

Not applicable.

\section{Competing interests}

The authors declare that they have no competing interests.

\section{References}

1. Ferlay J, Soerjomataram I, Dikshit R, Eser S, Mathers C, Rebelo M, Parkin DM, Forman D and Bray F: Cancer incidence and mortality worldwide: Sources, methods and major patterns in GLOBOCAN 2012. Int J Cancer 136: E359-E386, 2015.

2. Blaser MJ and Parsonnet J: Parasitism by the 'slow' bacterium Helicobacter pylori leads to altered gastric homeostasis and neoplasia. J Clin Invest 94: 4-8, 1994.

3. Huang JQ, Sridhar S, Chen Y and Hunt RH: Meta-analysis of the relationship between Helicobacter pylori seropositivity and gastric cancer. Gastroenterology 114: 1169-1179, 1998.

4. Correa P: Human gastric carcinogenesis: A multistep and multifactorial process-first American cancer society award lecture on cancer epidemiology and prevention. Cancer Res 52: 6735-6740, 1992.

5. Crabtree JE: Gastric mucosal inflammatory responses to Helicobacter pylori. Aliment Pharmacol Ther 10 (Suppl 1): S29-S37, 1996. 
6. Bartel DP: MicroRNAs: Genomics, biogenesis, mechanism, and function. Cell 116: 281-297, 2004.

7. He L and Hannon GJ: MicroRNAs: Small RNAs with a big role in gene regulation. Nat Rev Genet 5: 522-531, 2004.

8. Paladini L, Fabris L, Bottai G, Raschioni C, Calin GA and Santarpia L: Targeting microRNAs as key modulators of tumor immune response. J Exp Clin Cancer Res 35: 103, 2016.

9. Nakamura K, Sawada K, Yoshimura A, Kinose Y, Nakatsuka E and Kimura T: Clinical relevance of circulating cell-free microRNAs in ovarian cancer. Mol Cancer 15: 48, 2016.

10. Li X, Nie J, Mei Q and Han WD: MicroRNAs: Novel immunotherapeutic targets in colorectal carcinoma. World $\mathrm{J}$ Gastroenterol 22: 5317-5331, 2016.

11. Tsai MM, Wang CS, Tsai CY, Huang HW, Chi HC, Lin YH, Lu PH and Lin KH: Potential diagnostic, prognostic and therapeutic targets of MicroRNAs in human gastric cancer. Int J Mol Sci 17: E945, 2016.

12. Mishra PJ and Bertino JR: MicroRNA polymorphisms: The future of pharmacogenomics, molecular epidemiology and individualized medicine. Pharmacogenomics 10: 399-416, 2009.

13. Duan R, Pak C and Jin P: Single nucleotide polymorphism associated with mature miR-125a alters the processing of pri-miRNA. Hum Mol Genet 16: 1124-1131, 2007.

14. Arisawa T, Tahara T, Shibata T, Nagasaka M, Nakamura M, Kamiya Y, Fujita H, Hasegawa S, Takagi T, Wang FY, et al: A polymorphism of microRNA 27a genome region is associated with the development of gastric mucosal atrophy in Japanese male subjects. Dig Dis Sci 52: 1691-1697, 2007.

15. Dixon MF, Genta RM, Yardley JH and Correa P: Classification and grading of gastritis: The updated Sydney system. Am J Surg Pathol 20: 1161-1181, 1996.

16. Livak KJ and Schmittgen TD: Analysis of relative gene expression data using real-time quantitative PCR and the 2(-Delta Delta C(T)) method. Methods 25: 402-408, 2001.

17. Lim LP, Lau NC, Garrett-Engele P, Grimson A, Schelter JM, Castle J, Bartel DP, Linsley PS and Johnson JM: Microarray analysis shows that some microRNAs downregulate large numbers of target mRNAs. Nature 433: 769-773, 2005.

18. Chang L, Guo F, Huo B, Lv Y, Wang Y and Liu W: Expression and clinical significance of the microRNA-200 family in gastric cancer. Oncol Lett 9: 2317-2324, 2015.

19. Zhu P, Ahang J, Zhu J, Shi J, Zhu Q and Gao Y: miR-429 induces gastric carcinoma cell apoptosis through Bcl-2. Cell Physiol Biochem 37: 1572-1580, 2015.

20. Zhang M, Dong BB, Lu M, Zheng MJ, Chen H, Ding JZ, Xu AM and Xu YH: miR-429 functions as a tumor suppressor by targeting FSCN1 in gastric cancer cells. Onco Targets Ther 9: $1123-1133,2016$

21. Sun Y, Shen S, Liu X, Tang H, Wang Z, Yu Z, Li X and Wu M: miR-429 inhibits cells growth and invasion and regulates EMT-related marker genes by targeting Onecut 2 in colorectal carcinoma. Mol Cell Biochem 390: 19-30, 2014.
22. Ye ZB, Ma G, Zhao YH, Xiao Y, Zhan Y, Jing C, Gao K, Liu ZH and Yu SJ: miR-429 inhibits migration and invasion of breast cancer cells in vitro. Int J Oncol 46: 531-538, 2015.

23. Lei W, Liu YE, Zheng Y and Qu L: miR-429 inhibits ora squamous cell carcinoma growth by targeting ZEB1. Med Sci Monit 21: 383-389, 2015.

24. Chen Z, Liu X, Hu Z, Wang Y, Liu M, Liu X, Li H, Ji R, Guo Q and Zhou Y: Identification and characterization of tumor suppressor and oncogenic miRNAs in gastric cancer. Oncol Lett 10: 329-336, 2015.

25. Nam EJ, Yoon H, Kim SW, Kim H, Kim YT, Kim JH, Kim JW and Kim S: MicroRNA expression profiles in serous ovarian carcinoma. Clin Cancer Res 14: 2690-2695, 2008.

26. Reddy MA, Jin W, Villeneuve L, Wang M, Lanting L, Todorov I, Kato M and Natarajan R: Pro-inflammatory role of microrna-200 in vascular smooth muscle cells from diabetic mice. Arterioscler Thromb Vasc Biol 32: 721-729, 2012.

27. Xiao J, Tang J, Chen Q, Tang D, Liu M, Luo M, Wang Y, Wang J, Zhao Z, Tang C, et al: miR-429 regulates alveolar macrophage inflammatory cytokine production and is involved in LPS-induced acute lung injury. Biochem J 471: 281-291, 2015.

28. Brabletz S and Brabletz T: The ZEB/miR-200 feedback loop-a motor of cellular plasticity in development and cancer? EMBO Rep 11: 670-677, 2010

29. Watanabe T, Goto H, Arisawa T, Hase S, Niwa Y, Hayakawa T and Asai J: Relationship between local immune response to Helicobacter pylori and the diversity of disease: Investigation of H.pylori-specific IgA in gastric juice. J Gastroenterol Hepatol 12: 660-665, 1997.

30. Nam KT, Lee HJ, Sousa JF, Weis VG, O'Neal RL, Finke PE, Romero-Gallo J, Shi G, Mills JC, Peek RM Jr, et al: Mature chief cells are cryptic progenitors for metaplasia in the stomach. Gastroenterology 139: 2028-2037.e9, 2010.

31. Fox JG, Blanco M, Murphy JC, Taylor NS, Lee A, Kabok Z and Pappo J: Local and systemic immune responses in murine Helicobacter felis active chronic gastritis. Infect Immun 61: 2309-2015, 1993.

32. Yoshizawa N, Takenaka Y, Yamaguchi H, Tetsuya T, Tanaka H, Tatematsu M, Nomura S, Goldenring JR and Kaminishi M: Emergence of spasmolytic polypeptide-expressing metaplasia in Mongolian gerbils infected with Helicobacter pylori. Lab Invest 87: 1265-1276, 2007.

33. Halldórsdóttir AM, Sigurdardóttrir M, Jónasson JG, Oddsdóttir M, Magnússon J, Lee JR and Goldenring JR: Spasmolytic polypeptide expressing metaplasia (SPEM) associated with gastric cancer in Iceland. Dig Dis Sci 48: 431-441, 2003. 